\title{
Early Observations on the Use of Riociguat in a Large, Metropolitan Pulmonary Arterial Hypertension/ Chronic Thromboembolic Pulmonary Hypertension Treatment Center
}

Roxana Sulica $\cdot$ Rebecca Fenton · Frank Cefali

To view enhanced content go to www.cardiologytherapy-open.com

Received: April 24, 2015 / Published online: September 28, 2015

(C) The Author(s) 2015. This article is published with open access at Springerlink.com

\section{ABSTRACT}

Introduction: Pulmonary arterial hypertension (PAH) and chronic thromboembolic pulmonary hypertension (CTEPH) are rare, life-threatening diseases in which chronically elevated pressure in the pulmonary arteries results in vascular remodeling and right heart failure. Treatment goals are to improve patient functioning, exercise capacity, and symptoms; delay disease progression; normalize the right ventricular function; and, ultimately, improve survival. Therapeutic management centers on the affected physiologic pathways and includes endothelin receptor antagonists, phosphodiesterase-5 inhibitors, and prostacyclins. Recently, riociguat, a novel therapeutic agent that stimulates soluble guanylate cyclase via the nitric oxide pathway, was approved for the treatment of both $\mathrm{PAH}$ and CTEPH. Clinical trial data show that

Electronic supplementary material The online version of this article (doi:10.1007/s40119-015-0046-y) contains supplementary material, which is available to authorized users.

R. Sulica $(\bowtie) \cdot$ R. Fenton · F. Cefali

Pulmonary Hypertension Program, Mount Sinai

Beth Israel, New York, NY, USA

e-mail: rsulica@chpnet.org riociguat significantly improves exercise capacity as well as hemodynamic parameters in PAH/CTEPH.

Methods: We report on the early use of riociguat at our center-a large, metropolitan pulmonary hypertension treatment facility that cares for $>250$ patients with PAH/CTEPH. Through our initial clinical experience, we offer evidence on the benefits of riociguat in three patients with PAH associated with different etiologies, symptoms, and treatment goals.

Results: Overall, patients at our center who have received riociguat have experienced clinical benefits, including improvement in symptomatic and hemodynamic parameters, increase in 6-min walk distance, and improvement or stabilization of World Health Organization functional class. In several cases, initial response to riociguat has been encouraging and has helped patients reach their treatment goals. Riociguat appears to be well tolerated, with only one patient experiencing mild, self-limiting side effects.

Conclusion: Novel agents are continuously being introduced into the PAH/CTEPH armamentarium, and clinicians must decide 
how best to integrate them into their existing treatment algorithms. This case series offers initial evidence from our practice on the benefits of riociguat in optimizing hemodynamic and functional parameters. These benefits have been observed in PAH associated with different etiologies and functional status, and in both first-line and combination use.

Funding: Bayer HealthCare Pharmaceuticals.

Keywords: Chronic thromboembolic pulmonary hypertension (CTEPH); Nitric oxide pathway; Pulmonary arterial hypertension (PAH); Riociguat; Soluble guanylate cyclase

\section{INTRODUCTION}

In patients with persistent, severe pulmonary hypertension, two important differentials to consider are pulmonary arterial hypertension (PAH) and chronic thromboembolic pulmonary hypertension (CTEPH). Both are rare, life-threatening diseases in which chronically elevated pressure in the pulmonary arteries results in vascular remodeling and right heart failure $[1,2]$.

$\mathrm{PAH}$ is considered idiopathic (IPAH) if all known associated and predisposing conditions have been ruled out. Non-IPAH disease may be heritable or caused by exposure to drugs or toxins. It is also associated with underlying comorbidities, such as connective tissue disease (CTD), certain heart conditions, portal hypertension, or human immunodeficiency virus [3].

The precise etiology of CTEPH is undefined, though the principle cause is believed to be unresolved pulmonary emboli. Patients with prothrombotic conditions, such as lupus and hereditary thrombophilia, may be at higher risk for CTEPH following a pulmonary embolism [2].

Hemodynamic testing in PAH/CTEPH reveals a mean pulmonary arterial pressure (mPAP) of $>25 \mathrm{mmHg}$ at rest, normal left-sided filling pressures (i.e., pulmonary artery wedge pressure or left ventricular end-diastolic pressure $\leq 15 \mathrm{mmHg}$ ), along with increased pulmonary vascular resistance (PVR $>3$ Wood units) [4]. In cases of impaired right ventricular (RV) function, there is also decreased pulmonary arterial (PA) saturation [5]. Elevations in N-terminal pro-brain natriuretic peptide (NT-proBNP), a biomarker of RV dysfunction, are also frequently observed. Symptomatically, patients may present with decreased exercise tolerance, dyspnea on exertion (DOE), syncope, chest pain, palpitations, and edema [5].

Treatment goals in PAH/CTEPH are to improve World Health Organization functional class (WHO FC), increase exercise capacity, normalize RV function, delay disease progression, and ultimately improve survival [6]. Historically, oral agents, such as endothelin receptor antagonists (ERAs) and/or phosphodiesterase-5 inhibitors (PDE-5is), have been first-line therapies for patients with a low-risk profile. Patients are offered add-on therapies for progressive disease. Prostacyclins are typically reserved for patients with a high-risk profile, advanced disease, or disease that has progressed after prior treatment [7]. Background therapies, such as anticoagulants, are important in CTEPH and may also be used in PAH $[2,8]$.

Riociguat is the first member of a novel class of therapeutics called soluble guanylate cyclase (sGC) stimulators [9-13]. Riociguat has a dual mode of action: it sensitizes sGC to endogenous nitric oxide (NO) by stabilizing NO-sGC binding and also directly stimulates sGC via a different binding site, independently of NO [11, 
12]. Riociguat restores the NO-sGC-cGMP pathway and leads to increased generation of cGMP [11, 12, 14] (Fig. 1). Results from animal studies have demonstrated that sGC stimulation and increases in cGMP lead to vasodilatory, antifibrotic, antiproliferative, and anti-inflammatory effects [10-12, 14-23].

In the large-scale, phase 3 PATENT-1 (Pulmonary Arterial Hypertension Soluble Guanylate Cyclase-Stimulator; ClincalTrials.gov identifier, NCT00810693) trial, riociguat significantly improved 6-min walk distance (6MWD) from baseline to week 12 compared with placebo $(+30 \mathrm{~m}$ vs. $-6 \mathrm{~m}$, respectively) in patients with symptomatic $\mathrm{PAH}$, as well as those pretreated with ERAs or (nonintravenous) prostanoids [24, 25]. Riociguat also significantly and consistently improved a range of secondary endpoints, including PVR, NT-proBNP, WHO FC, time to clinical worsening, and Borg dyspnea score [24].

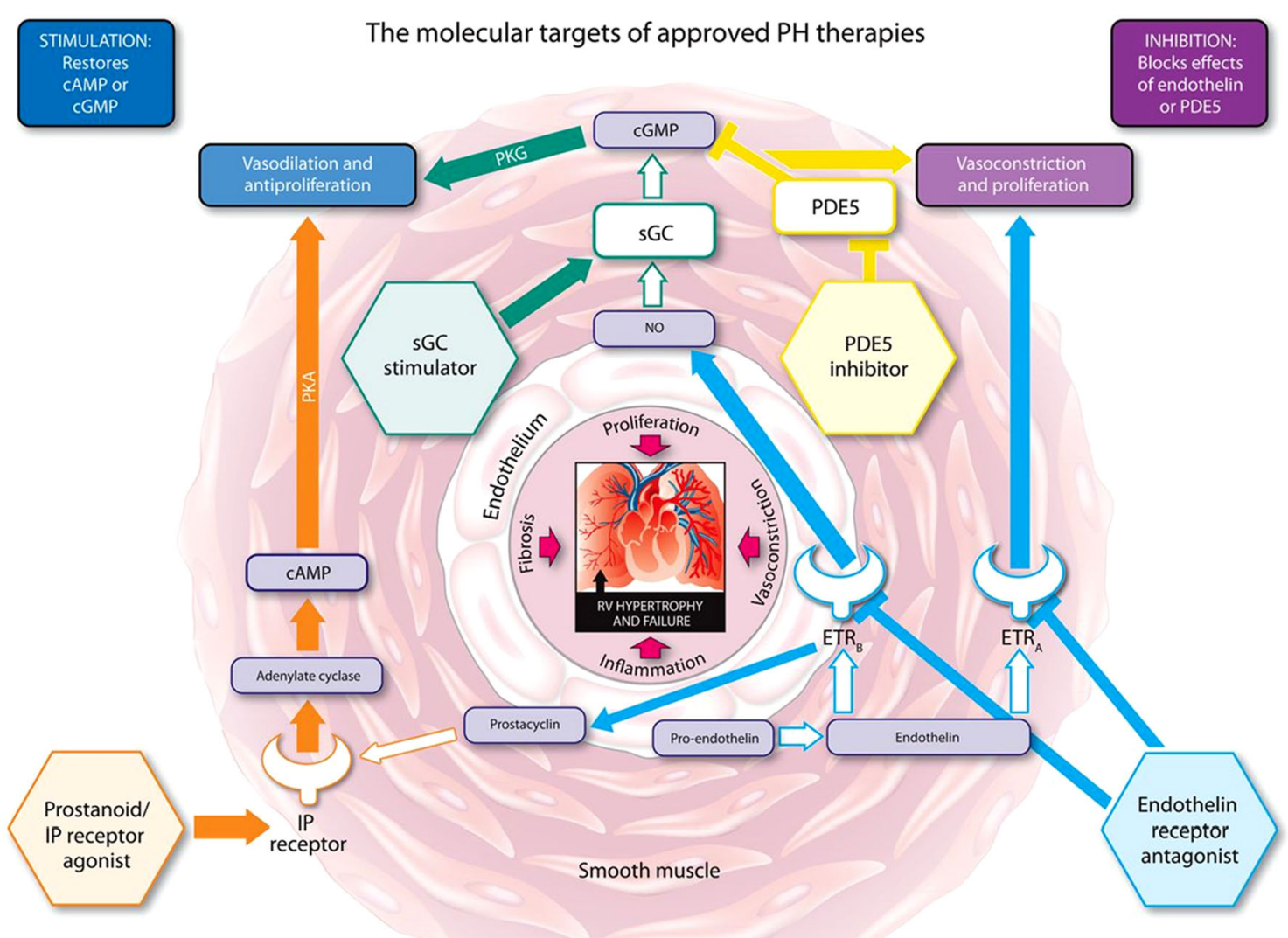

\begin{tabular}{|c|c|c|c|}
\hline KEY: & $\begin{array}{l}\text { Signalling pathway molecules } \\
\text { Target receptors }\end{array}$ & $\begin{array}{l}\text { PH therapies } \\
\text { Target enzymes }\end{array}$ & $\begin{array}{l}\text { Activation } \\
\text { Inhibitory action }\end{array}$ \\
\hline
\end{tabular}

Fig. 1 The molecular targets, signaling pathways, and modes of action of approved pulmonary hypertension $(\mathrm{PH})$ therapies. $c A M P$ cyclic adenosine monophosphate, $c G M P$ cyclic guanosine monophosphate, $E T R_{A}$ endothelin receptor $\mathrm{A}, E T R_{B}$ endothelin receptor $\mathrm{B}, I P$ prostacyclin, $N O$ nitric oxide, PDE5 phosphodiesterase type 5, PKA phosphate kinase A, $P K G$ cGMP-dependent protein kinase, $s G C$ soluble guanylate cyclase. Reprinted from Humbert and Ghofrani. Thorax. 2015; Thorax doi:10.1136/thoraxjnl2015-207170 [online first] via Creative Commons Open Access license: http://creativecommons.org/licenses/by-nc/4. $0 /$ legalcode 
Similar safety and efficacy results were seen in the phase 3 CHEST-1 (Chronic Thromboembolic Pulmonary Hypertension Guanylate Cyclase Stimulator Trial-1; ClinicalTrials.gov identifier, NCT00855465) trial in patients with CTEPH-where a 39-m increase in 6MWD from baseline to week 16 was seen in patients receiving riociguat compared with a decrease of $6 \mathrm{~m}$ in those receiving placebo. Riociguat also improved secondary endpoints, with decreases in PVR, NT-proBNP, WHO FC, and time to clinical worsening [26].

The following case series reports on the early use of riociguat at our center-a large pulmonary hypertension treatment facility in New York City (NY, USA). We care for $>250$ patients with $\mathrm{PAH} / \mathrm{CTEPH}$ and routinely use the full armamentarium of $\mathrm{PAH}$ therapies. Through our initial clinical experience, we offer evidence on the benefits of riociguat in three patients with $\mathrm{PAH}$ associated with different etiologies, symptoms, and treatment goals. All procedures followed were in accordance with the ethical standards of the responsible committee on human experimentation (institutional and national) and with the Helsinki Declaration of 1964, as revised in 2013. As this was a retrospective de-identified case series and no identifying details are discussed in this article, verbal consent only was obtained from all patients included.

\section{CASE PRESENTATIONS}

\section{Case 1}

\section{Referral Pathway}

A 44-year-old man with Sjögren's syndrome was seen at our center for pulmonary testing and evaluation in 2007. He was referred by his rheumatologist following abnormal echocardiographic findings, including right ventricle dilatation. Due to a history of underlying CTD, the suspicion for associated $\mathrm{PAH}(\mathrm{APAH})$ was high.

\section{Baseline Evaluation}

The initial diagnostic work-up included right heart catheterization (RHC), which revealed elevated PAP and decreased PA saturation. The patient complained of DOE and diminished exercise capacity. His 6MWD upon presentation was $212 \mathrm{~m}$. The patient was diagnosed with APAH related to a primary diagnosis of CTD (Sjögren's syndrome) and was classified as WHO FC III.

\section{Therapeutic Management}

The patient was initiated on an oral ERA (bosentan $62.5 \mathrm{mg}$ orally twice daily, increased after 1 month to $125 \mathrm{mg}$ orally twice daily, per protocol). An oral PDE-5i (tadalafil $40 \mathrm{mg}$ once daily) was later added due to marginal clinical response. Additional therapies included warfarin $10 \mathrm{mg}$ once daily and concomitant background medications specific to the management of Sjögren's syndrome.

The patient experienced clinical worsening over 3 years despite being maintained on his medications. In 2010, following deterioration to WHO FC IV status, IV epoprostenol (dose adjusted to $40 \mathrm{ng} / \mathrm{kg} / \mathrm{min}$ ) was added to his treatment regimen.

In 2011, RHC showed a decrease in mPAP to $40 \mathrm{mmHg}$ from $60 \mathrm{mmHg}$ while on triple therapy (bosentan, tadalafil, and epoprostenol) and cardiac index $(\mathrm{CI})$ increase from $2.33 \mathrm{~L} /$ $\mathrm{min} / \mathrm{m}^{2}$ to $2.68 \mathrm{~L} / \mathrm{min} / \mathrm{m}^{2}$. The patient was now evaluated as WHO FC II.

In February 2013, after two additional years of stability, the patient requested alternate PAH medications given the side effects of his current regimen-namely, lower extremity edema and nasal congestion. Our treatment team agreed 
with the request and once macitentan became available he was switched from bosentan to oral macitentan (at a dose of $10 \mathrm{mg}$ once daily).

\section{Therapeutic Response}

Following the switch to macitentan, the patient reported less nasal congestion and reduced peripheral edema. His 6MWD improved, increasing to $430 \mathrm{~m}$ from a baseline value of $410 \mathrm{~m}$ prior to the switch to macitentan; however, he still complained of reduced exercise tolerance. The decision was made to transition him from tadalafil. This decision was based on the favorable symptomatic results seen with riociguat at our center as well as the potential antifibrotic effects associated with riociguat $[9,10]$.

No protocol currently exists for transitioning patients from a PDE-5i, but as the concomitant use of riociguat and PDE-5i's is contraindicated we opted for a 2-day washout period from tadalafil. The patient was started on a riociguat dosage of $1 \mathrm{mg}$ three times daily, and titrated by $0.5 \mathrm{mg}$ every 2 weeks to the maximum dosage of $2.5 \mathrm{mg}$ three times daily. The patient was also maintained on his current doses of macitentan and IV epoprostenol.

Both the transition to riociguat and the titration to maximum therapeutic dosage were well tolerated. The patient initially reported moderate headaches (5/10 severity), successfully treated with oral acetaminophen. After 2 weeks, the headaches spontaneously resolved, and no further treatment was necessary.

Six months after starting therapy with riociguat, the patient's 6MWD increased to $480 \mathrm{~m}$ and FC has remained stable (WHO FC II). Subjectively, the patient reported significant improvement in his PAH symptoms, notably in the area of exercise tolerance. Further assessment showed, essentially, normalization of the patient's hemodynamics at 9-month follow-up on a triple combination of epoprostenol, macitentan, and riociguat-with a stable PA saturation of $69 \%$, decreased mPAP of $23 \mathrm{mmHg}$, increased CI of $3.13 \mathrm{~L} / \mathrm{min} / \mathrm{m}^{2}$, and decreased PVR of 2.71 Wood units.

\section{Case 2}

\section{Referral Pathway}

A 65-year-old woman with a 5-year history of scleroderma/CREST syndrome was first evaluated at our center in 2011. She was referred by her cardiologist after abnormal echocardiographic findings [resting RV systolic pressure (RVSP) of $71 \mathrm{~mm} \mathrm{Hg}$, and complaints of excessive fatigue, intermittent chest pressure, and palpitations. The patient's history of underlying CTD raised the possibility of an APAH diagnosis.

\section{Baseline Evaluation}

Baseline echocardiograph showed normal rightand left-ventricle size and function with mild mitral regurgitation and an RVSP of $47 \mathrm{~mm} \mathrm{Hg}$. RHC confirmed the diagnosis of APAH with an mPAP of $30 \mathrm{mmHg}$, PA wedge pressure of $8 \mathrm{mmHg}$, and $\mathrm{CI}$ of $2.83 \mathrm{~L} / \mathrm{min} / \mathrm{m}^{2}$. Her 6MWD was $213 \mathrm{~m}$. The patient was diagnosed with WHO FC Class III APAH related to scleroderma. Prior to initiation of therapy, she was lost to follow-up.

In 2014, the patient was readmitted with worsening symptoms characterized by decreased exercise capacity for 1 month, culminating in an inability to get out of bed. Further examination revealed atrial flutter, elevated liver enzymes, and acute kidney 
injury likely due to cardio-renal syndrome. After undergoing cardiac rate stabilization and hemodialysis repeat echocardiogram and RHC were performed. Results showed an increased mPAP of $35 \mathrm{mmHg}$, and a decreased PA saturation of $46 \%$, while echocardiogram showed RV dilatation, and hypokinesis. She was classified as having advanced WHO FC III $\mathrm{PAH}$.

\section{Therapeutic Management}

Riociguat was chosen as a first-line treatment based on its therapeutic efficacy $[10,11]$ and potential antifibrotic effects [11]. An ERA was not chosen due to the potential for edema/fluid overload and further liver enzyme elevation. The patient was started on riociguat $1 \mathrm{mg}$ three times daily and titrated to $2.0 \mathrm{mg}$ three times daily.

\section{Therapeutic Response}

The patient returned for evaluation 4 weeks after starting riociguat, at which time her 6MWD had increased to $365 \mathrm{~m}$. She reported improvement in DOE allowing her to climb five flights of stairs when her building's elevator was not working. This rapid functional improvement was impressive given the $2.0 \mathrm{mg}$ three times daily dose-less than the maximal dose of $2.5 \mathrm{mg}$. The patient denied experiencing any side effects from therapy with riociguat.

The patient returned for follow-up evaluation after 10 weeks and stated that her exercise capacity had continued to improve. Echocardiography revealed normal RV size and function. Although the patient's RVSP had increased to $65 \mathrm{mmHg}$, we considered the normalization of $\mathrm{RV}$ function to be a better indicator of therapeutic response. Her 6MWD was maintained at $365 \mathrm{~m}$. Her symptoms and exercise tolerance were consistent with FC improvement to WHO II.

\section{Case 3}

\section{Referral Pathway}

A 51-year-old man was first evaluated at our center in 2013. He was referred by his primary medical doctor based on echocardiographic findings of severe RA and RV dilatation and severe RV dysfunction, septal flattening, and symptomatic complaints of progressive DOE associated with palpitations that were relieved by rest.

\section{Baseline Evaluation}

Baseline echocardiograph showed septal flattening consistent with RV pressure overload; RHC confirmed the presence of severe $\mathrm{PAH}$ associated with significant RV dysfunction. The patient's NT-proBNP level was $385 \mathrm{pg} / \mathrm{mL}$ and his 6MWD was $426 \mathrm{~m}$.

The patient was diagnosed with WHO FC III IPAH. Although his 6MWD was favorable, his low CI $\left(1.66 \mathrm{~L} / \mathrm{min} / \mathrm{m}^{2}\right)$, relatively low PA saturation $(52 \%)$, and echocardiographic stigmata of poor prognosis prompted our team to offer continuous parenteral prostacyclin therapy, which he adamantly refused. As an alternative, he was started on combination therapy in rapid succession.

\section{Therapeutic Management}

The decision was made to first start the patient on an ERA. Macitentan was selected given its favorable long-term data on morbidity and mortality [27]. The patient was started on a dose of $10 \mathrm{mg}$ once daily, along with background therapy of furosemide $40 \mathrm{mg}$ once daily and potassium chloride $20 \mathrm{mEq}$ once daily.

\section{Therapeutic Response}

The patient returned 2 weeks after starting macitentan. He reported mild symptomatic 
improvement, including greater energy and decreased DOE. At 6 weeks, his 6MWD had increased slightly to $457 \mathrm{~m}$ from a baseline of $426 \mathrm{~m}$ and his FC remained unchanged. While his NT-proBNP level had decreased to $183 \mathrm{pg} /$ $\mathrm{mL}$, it was still nearly double the upper limits of normal (limits of normal 0-100 pg/mL).

Riociguat was then added to the treatment regimen based on its clinical efficacy data [9] and its theoretical mechanistic advantage over PDE-5is in this patient with chronically elevated NT-proBNP and persistent FC III symptoms $[10,11]$. The patient was started on riociguat $1 \mathrm{mg}$ three times daily and dose adjusted to the maximum therapeutic dosage of $2.5 \mathrm{mg}$ three times daily.

During the 6-month evaluation on combination therapy, the patient reported more energy and less DOE. His symptoms were classified as FC II and his 6MWD increased to $495 \mathrm{~m}$. NT-proBNP levels at his 6-month follow-up showed normalization to $31.5 \mathrm{pg} /$ $\mathrm{mL}$. The patient denied any side effects from therapy with riociguat.

After 9 months of combination therapy, repeat echocardiographic still showed RV dilatation, but only a mild-moderate degree of RV dysfunction. A repeat RHC 1 month later confirmed improved RV function, as well as an increase in PA saturation to $64 \%$ and an increase in CI to $2.18 \mathrm{~L} / \mathrm{min} / \mathrm{m}^{2}$.

The patient is responding well to combination therapy, with subjective and objective improvement noted since the addition of riociguat. Given his continued RV dysfunction and a relatively low CI, our team is considering the addition of inhaled or oral prostacyclin therapy.

\section{DISCUSSION}

Recent clinical guidelines from the European Society of Cardiology (ESC) and the European Respiratory Society (ERS) have included riociguat as initial therapy with grade IB recommendation for patients with WHO FC II-III PAH [28]. Additional consensus-based guidelines recommended riociguat as monotherapy for treatment-naïve WHO FC II-III patients (grade CB) and as add-on therapy in WHO FC III-IV patients currently on stable doses of ERAs or inhaled prostanoids (grade CB) [29]. For WHO FC III patients whose clinical status is unacceptable despite PAH-monotherapy, addition of a second class of PAH therapy is warranted. No algorithm currently exists for its use in the treatment of CTEPH. Limited guidance is available on agent-specific $\mathrm{PAH}$ combination therapy due to a lack of comparative effectiveness data [28, 29].

All three patients reported in our series' presented as WHO FC III for which monotherapy with an ERA, PDE-5i, or sGC stimulator is recommended.

There are no formal agent-specific guidelines for add on or switching therapies and as such, evidence-based guidance was lacking for transition from a PDE-5i to riociguat while simultaneously receiving macitentan and IV epoprostenol. Based on the pharmacokinetics of tadalafil, a 2-day washout period was chosen and riociguat was successfully titrated [30]. Riociguat was chosen over tadalafil based on the antifibrotic properties it has exhibited in preclinical studies and their potential benefit in a patient presenting with concomitant PAH and 
CTD [21-23]. The first patient had a remarkable therapeutic response of hemodynamic normalization on triple therapy that included intravenous epoprostenol. However, hemodynamic normalization was only achieved after bosentan was changed to a newer generation ERA, and tadalafil switched to riociguat. The effect of macitentan-riociguat combination therapy was not examined in the PATENT-1 trial (where the ERA used for background therapy was bosentan).

There are currently no effective means of determining which patients may benefit from specific agents, and as such, familiarity with individual patient history is critical when managing advanced disease and establishing optimal pharmacotherapy for PAH $[28,29]$. Our experience with a WHO FC III patient with underlying CTD, edema, and liver enzyme elevation suggests that riociguat represents an important first-line option for patients with an aggressive disease course who are not appropriate for ERAs due to risks of adverse events. However, it is critical that these patients be evaluated at an experienced center capable of diagnosing and managing patients with $\mathrm{PAH}$ [28, 29].

New large-scale trials have produced data on both long-term outcomes and the safety and efficacy of new combination regimens. Historically, PAH medications have been used in combination in an add-on fashion, as dictated by the goal-directed therapy principle: therapy is reassessed every 3 to 6 months and new treatment agents are added if patients do not achieve certain clinical and hemodynamic goals [8]. However, in the modern era, this approach may change to concomitant combination therapy upfront from the moment of diagnosis, as supported by recently published findings from the large-scale AMBITION study (A Randomized,
Double-Blind, Multicenter Study of First-Line Combination Therapy With AMBrIsentan and Tadalafil in Patients With Pulmonary Arterial HypertensION; ClinicalTrials.gov identifier, NCT01178073) and recommended in the recently updated ESC/ERS guidelines [28]. The AMBITION study has shown that first-line combination therapy in $\mathrm{PAH}$ improves clinical outcomes over monotherapy [31].

It has been our general practice to be assertive with early combination therapy in patients with severe PAH as evidenced by our decision to initiate macitentan and riociguat combination therapy in a patient who refused parenteral prostacyclin therapy. As his PAH was rapidly progressing, riociguat was chosen over a PDE-5i due to its novel action on the NO pathway $[11,12]$. To date, the rapid, sequential addition of riociguat to an ERA to enhance treatment response has not been studied in clinical trials. However, we offer evidence that a patient with New York Heart Association (NYHA) FC III symptoms can achieve significant functional and hemodynamic improvements with this regimen, despite evidence of severe $\mathrm{PAH}$ disease on echocardiogram and RHC. As the patient ultimately showed significant improvement, we feel aggressive oral combination therapy may be suitable for certain patients provided close follow-up and monitoring.

\section{CONCLUSION}

Riociguat, a novel agent that acts via the NO pathway, was approved in the United States in 2013 for the treatment of both PAH and CTEPH. As a large, metropolitan $\mathrm{PAH} / \mathrm{CTEPH}$ treatment center actively involved in research, our site was an early adopter of riociguat. This case series offers initial evidence from our practice on 
benefits of riociguat in optimizing hemodynamic and functional parameters. Importantly, these benefits have been observed in PAH associated with different etiologies and functional status, as well as in both first-line use and combination use.

\section{ACKNOWLEDGMENTS}

Article processing charges for this article were funded by Bayer HealthCare Pharmaceuticals, Whippany, NJ, USA. Medical writing and editing assistance were provided by Katrina Rodies, CRNP, and Patricia Abramo of Adelphi Communications. This assistance was funded by Bayer HealthCare Pharmaceuticals. All named authors meet the International Committee of Medical Journal Editors (ICMJE) criteria for authorship for this manuscript, take responsibility for the integrity of the work as a whole, and have given final approval to the version to be published.

Disclosures. Dr. Roxana Sulica declares having received research grants from Actelion Pharmaceuticals Inc., Bellerophon Therapeutics, and Lung Biotechnology, and having served on advisory boards for Bayer HealthCare Pharmaceuticals, Gilead Sciences, Inc., and United Therapeutics Corporation. Ms. Rebecca Fenton and Mr. Frank Cefali have nothing to disclose.

Compliance with ethics guidelines. All procedures followed were in accordance with the ethical standards of the responsible committee on human experimentation (institutional and national) and with the Helsinki Declaration of 1964, as revised in 2013. As this was a retrospective de-identified case series and no identifying details are discussed in this article, verbal consent only was obtained from all patients included.

Open Access. This article is distributed under the terms of the Creative Commons Attribution-NonCommercial 4.0 International License (http://creativecommons.org/licenses/bync/4.0/), which permits any noncommercial use, distribution, and reproduction in any medium, provided you give appropriate credit to the original author(s) and the source, provide a link to the Creative Commons license, and indicate if changes were made.

\section{REFERENCES}

1. Badesch DB, Abman SH, Simonneau G, Rubin LJ, McLaughlin VV. Medical therapy for pulmonary arterial hypertension: updated ACCP evidence-based clinical practice guidelines. Chest. 2007;131(6):1917-28.

2. McNeil K, Dunning J. Chronic thromboembolic pulmonary hypertension (CTEPH). Heart. 2007;93(9):1152-8.

3. Simonneau G, Gatzoulis MA, Adatia I, et al. Updated clinical classification of pulmonary hypertension. J Am Coll Cardiol. 2013;62(1 suppl):D34-41.

4. McLaughlin VV, McGoon MD. Pulmonary arterial hypertension. Circulation. 2006;114(13):1417-31.

5. Barst RJ, McGoon M, Torbicki A, et al. Diagnosis and differential assessment of pulmonary arterial hypertension. J Am Coll Cardiol. 2004;43((12 suppl S)):40S-7S.

6. McLaughlin VV, Gaine SP, Howard LS, et al. Treatment goals of pulmonary hypertension. J Am Coll Cardiol. 2013;62((25 suppl D)):D73-81.

7. Ruan C-H, Dixon RAF, Willerson JT, et al. Prostacyclin therapy for pulmonary arterial hypertension. Tex Heart Inst J. 2010;37(4):391-9.

8. Galiè N, Corris PA, Frost A, et al. Updated treatment algorithm of pulmonary arterial hypertension. J Am Coll Cardiol. 2013;62((25 suppl D)):D60-72.

9. Adempas $^{\circledR}$ [package insert]. Whippany: Bayer HealthCare Pharmaceuticals Inc; 2014. 
10. Schermuly RT, Stasch J-P, Pullamsetti SS, et al. Expression and function of soluble guanylate cyclase in pulmonary arterial hypertension. Eur Respir J. 2008;32(4):881-91.

11. Stasch J-P, Pacher P, Evgenov OV. Soluble guanylate cyclase as an emerging therapeutic target in cardiopulmonary disease. Circulation. 2011;123(20):2263-73.

12. Stasch J-P, Evgenov OV. Soluble guanylate cyclase stimulators in pulmonary hypertension. Handb Exp Pharmacol. 2013;218:279-313.

13. Follmann M, Griebenow N, Hahn MG, et al. The chemistry and biology of soluble guanylate cyclase stimulators and activators. Angew Chem Int Ed Engl. 2013;52(36):9442-62.

14. Stasch J-P, Hobbs AJ. NO-independent, haem-dependent soluble guanylate cyclase stimulators. Handb Exp Pharmacol. 2009;191:277-308.

15. Dumitrascu R, Weissmann N, Ghofrani HA, et al. Activation of soluble guanylate cyclase reverses experimental pulmonary hypertension and vascular remodeling. Circulation. 2006;113(2):286-95.

16. Geschka S, Kretschmer A, Sharkovska Y, et al. Soluble guanylate cyclase stimulation prevents fibrotic tissue remodeling and improves survival in salt-sensitive Dahl rats. PLoS One. 2011;6(7):e21853.

17. Lang M, Kojonazarov B, Tian X, et al. The soluble guanylate cyclase stimulator riociguat ameliorates pulmonary hypertension induced by hypoxia and SU5416 in rats. PLoS One. 2012;7(8):e43433.

18. Sharkovska Y, Kalk P, Lawrenz B, et al. Nitric oxide-independent stimulation of soluble guanylate cyclase reduces organ damage in experimental low-renin and high-renin models. J Hypertens. 2010;28(8):1666-75.

19. Evgenov OV, Zou L, Zhang M, et al. Stimulation of soluble guanylate cyclase attenuates bleomycin-induced pulmonary fibrosis in mice. Am J Respir Crit Care Med. 2011;183:A2715.

20. Becker EM, Stasch J-P, Bechem M, et al. Effects of different pulmonary vasodilators on arterial saturation in a model of pulmonary hypertension. PLoS One. 2013;8(8):e73502.
21. Beyer C, Reich N, Schindler SC, et al. Stimulation of soluble guanylate cyclase reduces experimental dermal fibrosis. Ann Rheum Dis. 2012;71:1019-26.

22. Beyer C, Zenzmaier C, Palumbo-Zerr K, et al. Stimulation of the soluble guanylate cyclase (sGC) inhibits fibrosis by blocking non-canonical TGFb signalling. Ann Rheum Dis. 2015;74(7):1408-16.

23. Dees C, Beyer C, Distler A, et al. Stimulators of soluble guanylate cyclase (sGC) inhibit experimental skin fibrosis of different aetiologies. Ann Rheum Dis. 2015;74(8):1621-5.

24. Ghofrani H-A, Galiè N, Grimminger F, for the PATENT-1 Study Group, et al. Riociguat for the treatment of pulmonary arterial hypertension. N Engl J Med. 2013;369(4):330-40.

25. Humbert MJC, Galie N, Ghofrani HA, et al. Efficacy of riociguat in pretreated versus treatment-naïve patients with pulmonary arterial hypertension (PAH) in the phase III PATENT-1 study. Am J Respir Crit Care Med. 2013;187:A3534.

26. Ghofrani H-A, D'Armini AM, Grimminger F, et al. Riociguat for the treatment of chronic thromboembolic pulmonary hypertension. N Engl J Med. 2013;369:319-29.

27. Pulido T, Adzerikho I, Channick RN, for SERAPHIN Investigators, et al. Macitentan and morbidity and mortality in pulmonary arterial hypertension. N Engl J Med. 2013;369(9):809-18.

28. Galie N, Humbert M, Vachiery J-L, et al. 2015 ESC/ ERS guidelines for the diagnosis and treatment of pulmonary hypertension: the joint task force for the diagnosis and treatment of pulmonary hypertension of the European Society of Cardiology (ESC) and the European Respiratory Society (ERS). Eur Heart J. 2015. doi:10.1093/ eurheartj/ehv317 [Epub ahead of print]

29. Taichman DB, Ornelas J, Chung L, et al. Pharmacologic therapy for pulmonary hypertension in adults. CHEST guidelines and expert panel report. Chest. 2014;146:449-75.

30. Forgue ST, Patterson BE, Bedding AW, et al. Tadalafil pharmacokinetics in healthy subjects. Br J Clin Pharmacol. 2006;61:280-8.

31. Galie N, Barbera JA, Frost A, et al. Initial use of ambrisentan plus tadalafil in pulmonary arterial hypertension. New Engl J Med. 2015;373:834-44. 